\title{
The Prophylactic Use of Antibiotics in Surgery
}

\author{
Voros DC
}

Almost 50 years have passed since the publication of the now historic studies by Polk (1969), Nichols (1973), Stone (1976) and Condon (1979) [1-4], on the efficacy of perioperative antibiotic use for the prevention of surgical site infections (SSIs, infections of the operative incision and/or operative space) in elective surgery. Subsequent reports by the same authors, as well as by other surgeons and infectious disease specialists, largely confirmed the original findings, thus rendering perioperative antibiotic use an integral part of the contemporary standard of care, as described in clinical practice guidelines published by multiple scientific societies, national and international health organizations $[5,6]$.

The principal message of the aforementioned studies was, and continues to be, that while appropriate skin antisepsis and good operative technique (particularly with respect to limiting tissue damage and hematoma formation and avoiding the use of non-functioning drains) are important in preventing SSIs, the perioperative use of antibiotics can reduce the incidence of such infectious complications still further. In order to optimize outcomes, it is critical that antibiotic use for this indication should adhere to the following general principles: a) antibiotic(s) should be administered intravenously just before the operation and a repeat dose should be given only during certain lengthy and complex operations, b) the specific antibiotic(s) used should be tailored to the flora of the operated organ, the flora of the skin (when prosthetic materials are used) and the flora of the nosocomial environment, following consultation with the infection control committee of the hospital, c) provided that the aforementioned 'target' flora are covered, the antimicrobial spectrum of the antibiotic(s) used must be as narrow as possible, $d$ ) advanced, broad spectrum antibiotics should be reserved solely for therapeutic use, in the event that an infection develops after surgery. This principle not only ensures that a 'second line of defense' against postoperative infection is maintained, but also helps to arrest the extension of antimicrobial resistance,

Voros DC

Emeritus Professor of Surgery, University of Athens

Corresponding author: Voros C Dionysios Emeritus Professor of Surgery, University of Athens e-mail: diovoros@med.uoa.gr,d.voros@dunant.gr which has far-reaching negative implications for both the treated patient and others that are or will be hospitalized in the same hospital.

Unfortunately, the aforementioned principles are not always followed in practice, as surgeons may inappropriately employ advanced, broad spectrum antibiotics and/or administer multiple antibiotic doses for the prevention of SSIs. These practices likely stem from misplaced anxiety with respect to the outcome of the operation or from an erroneous belief that liberal antibiotic use may prevent the development of complications, which is far from the case. Inappropriate antibiotic use not only offers no immediate benefit to the patient, but, as discussed above, it also decreases the therapeutic options available in the case of postoperative infection and contributes to the emergence of resistant microorganisms in the hospital environment.

It is known that antibiotics are the only drugs with the potential to affect not only the patient receiving them, but also both present and future hospitalized patients. As such, surgeons should always keep in mind that clinical judgement should be firmly grounded on evidence, and that the duty to provide the best possible care to current patients does not provide justification for compromising the outcome of future patients. As is often the case in medicine, when it comes to the prophylaxis of SSIs, less is more.

\section{References}

1. Polk HC, Lopez-Mayor JF. Postoperative wound infection: A prospective study of determinant factors and prevention. Surgery 1969;66:97-103.

2. Nichols RL, Broido P, Condon RE, et al. Effect of preoperative neomycin-erythromycin intestinal preparation on the incidence of infectious complications following colon surgery. Ann Surg 1973;178:453.

3. Stone HH, Hooper AC, Kolb LD, et al. Antibiotic prophylaxis in gastric, biliary and colonic surgery. Ann Surg 1976;184Q:443-50.

4. Condon RE, Bartlett JG, Nichols RL, et al. Ochi S Pre-operative prophylactic cephalothin fails to control septic complications of colorectal operations: Results of a controlled clinical trial. Am J Surg 1979;137:68-74.

5. Pang Y Young, Rachel G Khadaroo. Surgical Site Infections, Surgical Clinics of North America 2014;94:1245-64.

6. Gilbert DN, Champers HF, Eliopoulos GM, et al. The Sanford Guide to Antimicrobial Therapy 2015. 45th ed; 2015. 